\title{
Error bounds for linear complementarity problems of weakly chained diagonally dominant $B$-matrices
}

Feng Wang*

\section{${ }^{\text {*Correspondence: }}$} wangf991@163.com College of Science, Guizhou Minzu University, Guiyang, Guizhou 550025, P.R. China

\begin{abstract}
In this paper, new error bounds for the linear complementarity problem are obtained when the involved matrix is a weakly chained diagonally dominant $B$-matrix. The proposed error bounds are better than some existing results. The advantages of the results obtained are illustrated by numerical examples.
\end{abstract}

MSC: $90 \mathrm{C} 33 ; 60 \mathrm{G} 50 ; 65 \mathrm{~F} 35$

Keywords: error bound; linear complementarity problem; weakly chained diagonally dominant matrix; $B$-matrix

\section{Introduction}

A linear complementarity problem $(L C P)$ is to find a vector $x \in \mathbb{R}^{n \times 1}$ such that

$$
(M x+q)^{T} x=0, \quad M x+q \geq 0, \quad x \geq 0,
$$

where $M=\left[m_{i j}\right] \in \mathbb{R}^{n \times n}$ and $q \in \mathbb{R}^{n \times 1}$. The $L C P$ has various applications in the free boundary problems for journal bearing, the contact problem, and the Nash equilibrium point of a bimatrix game [1-3].

The $L C P$ has a unique solution for any $q \in \mathbb{R}^{n \times 1}$ if and only if $M$ is a $P$-matrix [4]. In [5], Chen $e t$ al. gave the following error bound for the $L C P$ when $M$ is a $P$-matrix:

$$
\left\|x-x^{*}\right\|_{\infty} \leq \max _{d \in[0,1]^{n}}\left\|(I-D+D M)^{-1}\right\|_{\infty}\|r(x)\|_{\infty}
$$

where $x^{*}$ is the solution of the $L C P, r(x)=\min \{x, M x+q\}, D=\operatorname{diag}\left(d_{i}\right)$ with $0 \leq d_{i} \leq 1$, and the min operator $r(x)$ denotes the componentwise minimum of two vectors. If $M$ satisfies special structures, then some bounds of $\max _{d \in[0,1]^{n}}\left\|(I-D+D M)^{-1}\right\|_{\infty}$ can be derived [6$11]$.

Definition 1 ([4]) A matrix $M=\left[m_{i j}\right] \in \mathbb{R}^{n \times n}$ is called a $B$-matrix if for any $i, j \in \mathbb{N}=$ $\{1,2, \ldots, n\}$,

$$
\sum_{k \in N} m_{i k}>0, \quad \frac{1}{n}\left(\sum_{k \in N} m_{i k}\right)>m_{i j}, \quad j \neq i
$$

(c) The Author(s) 2017. This article is distributed under the terms of the Creative Commons Attribution 4.0 International License (http://creativecommons.org/licenses/by/4.0/), which permits unrestricted use, distribution, and reproduction in any medium, provided you give appropriate credit to the original author(s) and the source, provide a link to the Creative Commons license, and indicate if changes were made. 
Definition 2 ([12]) A matrix $A=\left[a_{i j}\right] \in \mathbb{R}^{n \times n}$ is called a weakly chained diagonally dominant ( $w c d d)$ matrix if $A$ is diagonally dominant, i.e.,

$$
\left|a_{i i}\right| \geq r_{i}(A)=\sum_{j=1, \neq i}^{n}\left|a_{i j}\right|, \quad \forall i \in \mathbb{N},
$$

and for each $i \notin J(A)=\left\{i \in \mathbb{N}:\left|a_{i i}\right|>r_{i}(A)\right\} \neq \emptyset$, there is a sequence of nonzero elements of $A$ of the form $a_{i i_{1}}, a_{i_{1} i_{2}}, \ldots, a_{i_{r} j}$ with $j \in J(A)$.

Definition 3 ([13]) A matrix $M=\left[m_{i j}\right] \in \mathbb{R}^{n \times n}$ is called a weakly chained diagonally dominant $(w c d d) B$-matrix if it can be written in the form $M=B^{+}+C$ with $B^{+}$a $w c d d$ matrix whose diagonal entries are all positive.

García-Esnaola et al. [8] gave the upper bound for $\max _{d \in[0,1]^{n}}\left\|(I-D+D M)^{-1}\right\|_{\infty}$ when $M$ is a $B$-matrix: Let $M=\left[m_{i j}\right] \in \mathbb{R}^{n \times n}$ be a $B$-matrix with the form

$$
M=B^{+}+C,
$$

where

$$
B^{+}=\left[b_{i j}\right]=\left[\begin{array}{ccc}
m_{11}-r_{1}^{+} & \cdots & m_{1 n}-r_{1}^{+} \\
\vdots & & \vdots \\
m_{n 1}-r_{n}^{+} & \cdots & m_{n n}-r_{n}^{+}
\end{array}\right]
$$

and $r_{i}^{+}=\max \left\{0, m_{i j} \mid j \neq i\right\}$. Then

$$
\max _{d \in[0,1]^{n}}\left\|(I-D+D M)^{-1}\right\|_{\infty} \leq \frac{n-1}{\min \{\beta, 1\}},
$$

where $\beta=\min _{i \in \mathbb{N}}\left\{\beta_{i}\right\}$ and $\beta_{i}=b_{i i}-\sum_{j \neq i}\left|b_{i j}\right|$.

To improve the bound in (2), Li et al. [14] presented the following result: Let $M=\left[m_{i j}\right] \in$ $\mathbb{R}^{n \times n}$ be a $B$-matrix with the form $M=B^{+}+C$, where $B^{+}=\left[b_{i j}\right]$ is defined as (1). Then

$$
\max _{d \in[0,1]^{n}}\left\|(I-D+D M)^{-1}\right\|_{\infty} \leq \sum_{i=1}^{n} \frac{n-1}{\min \left\{\bar{\beta}_{i}, 1\right\}} \prod_{j=1}^{i-1}\left(1+\frac{1}{\bar{\beta}_{j}} \sum_{k=j+1}^{n}\left|b_{j k}\right|\right)
$$

where $\bar{\beta}_{i}=b_{i i}-\sum_{j=i+1}^{n}\left|b_{i j}\right| l_{i}\left(B^{+}\right), l_{k}\left(B^{+}\right)=\max _{k \leq i \leq n}\left\{\frac{1}{\left|b_{i i}\right|} \sum_{j=k, \neq i}^{n}\left|b_{i j}\right|\right\}$ and

$$
\prod_{j=1}^{i-1}\left(1+\frac{1}{\bar{\beta}_{j}} \sum_{k=j+1}^{n}\left|b_{j k}\right|\right)=1, \quad \text { if } i=1
$$

Recently, when $M$ is a weakly chained diagonally dominant ( $w c d d) B$-matrix, Li et al. [13] gave a bound for $\max _{d \in[0,1]^{n}}\left\|(I-D+D M)^{-1}\right\|_{\infty}$ : Let $M=\left[m_{i j}\right] \in \mathbb{R}^{n \times n}$ be a $w c d d B$-matrix with the form $M=B^{+}+C$, where $B^{+}=\left[b_{i j}\right]$ is defined as (1). Then

$$
\max _{d \in[0,1]^{n}}\left\|(I-D+D M)^{-1}\right\|_{\infty} \leq \sum_{i=1}^{n}\left(\frac{n-1}{\min \left\{\tilde{\beta}_{i}, 1\right\}} \prod_{j=1}^{i-1} \frac{b_{j j}}{\tilde{\beta}_{j}}\right)
$$


where $\tilde{\beta}_{i}=b_{i i}-\sum_{j=i+1}^{n}\left|b_{i j}\right|>0$ and $\prod_{j=1}^{i-1}{\underline{\beta_{i j}}}_{\tilde{\beta}_{j}}=1$ if $i=1$.

This bound in (4) holds when $M$ is a $B$-matrix since a $B$-matrix is a weakly chained diagonally dominant $B$-matrix [13].

Now, some notation is given, which will be used in the sequel. Let $A=\left[a_{i j}\right] \in \mathbb{R}^{n \times n}$. For $i, j, k \in \mathbb{N}$, denote

$$
\begin{aligned}
& u_{i}(A)=\frac{1}{\left|a_{i i}\right|} \sum_{j=i+1}^{n}\left|a_{i j}\right|, \quad u_{n}(A)=0, \\
& b_{k}(A)=\max _{k+1 \leq i \leq n}\left\{\frac{\sum_{j=k, \neq i}^{n}\left|a_{i j}\right|}{\left|a_{i i}\right|}\right\}, \quad b_{n}(A)=1, \\
& p_{k}(A)=\max _{k+1 \leq i \leq n}\left\{\frac{\left|a_{i k}\right|+\sum_{j=k+1, \neq i}^{n}\left|a_{i j}\right| b_{k}(A)}{\left|a_{i i}\right|}\right\}, \quad p_{n}(A)=1 .
\end{aligned}
$$

The rest of this paper is organized as follows: In Section 2, we present some new bounds for $\max _{d \in[0,1]^{n}}\left\|(I-D+D M)^{-1}\right\|_{\infty}$ when $M$ is a wcdd $B$-matrix. Numerical examples are given to verify the corresponding results in Section 3.

\section{Main results}

In this section, some new upper bounds for $\max _{d \in[0,1]^{n}}\left\|(I-D+D M)^{-1}\right\|_{\infty}$ are provided when $M$ is a $w c d d B$-matrix. Firstly, several lemmas, which will be used later, are given.

Lemma 1 ([13]) Let $M=\left[m_{i j}\right] \in \mathbb{R}^{n \times n}$ be a wcdd B-matrix with the form $M=B^{+}+C$, where $B^{+}$is defined as (1). Then

$$
\left\|\left(I+\left(B_{D}^{+}\right)^{-1} C_{D}\right)^{-1}\right\|_{\infty} \leq n-1,
$$

where $B_{D}^{+}=I-D+D B^{+}$and $C_{D}=D C$.

Lemma 2 ([15]) Let $A=\left[a_{i j}\right] \in \mathbb{R}^{n \times n}$ be a wcdd M-matrix with $u_{k}(A) p_{k}(A)<1(\forall k \in \mathbb{N})$.

Then

$$
\begin{aligned}
\left\|A^{-1}\right\|_{\infty} \leq & \max \left\{\sum_{i=1}^{n}\left(\frac{1}{a_{i i}\left(1-u_{i}(A) p_{i}(A)\right)} \prod_{j=1}^{i-1} \frac{u_{j}(A)}{1-u_{j}(A) p_{j}(A)}\right),\right. \\
& \left.\sum_{i=1}^{n}\left(\frac{p_{i}(A)}{a_{i i}\left(1-u_{i}(A) p_{i}(A)\right)} \prod_{j=1}^{i-1} \frac{1}{1-u_{j}(A) p_{j}(A)}\right)\right\}
\end{aligned}
$$

where

$$
\prod_{j=1}^{i-1} \frac{u_{j}(A)}{1-u_{j}(A) p_{j}(A)}=1, \quad \prod_{j=1}^{i-1} \frac{1}{1-u_{j}(A) p_{j}(A)}=1, \quad \text { if } i=1 .
$$

Lemma 3 ([14]) Let $\gamma>0$ and $\eta \geq 0$. Then, for any $x \in[0,1]$,

$$
\frac{1}{1-x+\gamma x} \leq \frac{1}{\min \{\gamma, 1\}}, \quad \frac{\eta x}{1-x+\gamma x} \leq \frac{\eta}{\gamma} .
$$


Theorem 1 Let $M=\left[m_{i j}\right] \in \mathbb{R}^{n \times n}$ be a wcdd B-matrix with the form $M=B^{+}+C$, where $B^{+}=\left[b_{i j}\right]$ is defined as (1). If, for each $i \in \mathbb{N}$,

$$
\hat{\beta}_{i}=b_{i i}-\sum_{j=i+1}^{n}\left|b_{i j}\right| p_{i}\left(B^{+}\right)>0,
$$

then

$$
\begin{aligned}
& \max _{d \in[0,1]^{n}}\left\|(I-D+D M)^{-1}\right\|_{\infty} \\
& \quad \leq \max \left\{\sum_{i=1}^{n} \frac{n-1}{\min \left\{\hat{\beta}_{i}, 1\right\}} \prod_{j=1}^{i-1}\left(\frac{1}{\hat{\beta}_{j}} \sum_{k=j+1}^{n}\left|b_{j k}\right|\right), \sum_{i=1}^{n} \frac{(n-1) p_{i}\left(B^{+}\right)}{\min \left\{\hat{\beta}_{i}, 1\right\}} \prod_{j=1}^{i-1} \frac{b_{j j}}{\hat{\beta}_{j}}\right\},
\end{aligned}
$$

where

$$
\prod_{j=1}^{i-1}\left(\frac{1}{\hat{\beta}_{j}} \sum_{k=j+1}^{n}\left|b_{j k}\right|\right)=1, \quad \prod_{j=1}^{i-1} \frac{b_{j j}}{\hat{\beta}_{j}}=1, \quad \text { if } i=1 .
$$

Proof Let $M_{D}=I-D+D M$. Then

$$
M_{D}=I-D+D M=I-D+D\left(B^{+}+C\right)=B_{D}^{+}+C_{D}
$$

where $B_{D}^{+}=I-D+D B^{+}$. Similar to the proof of Theorem 2 in [13], we see that $B_{D}^{+}$is a $w c d d$ $M$-matrix with positive diagonal elements and $C_{D}=D C$, and, by Lemma 1 ,

$$
\left\|M_{D}^{-1}\right\|_{\infty} \leq\left\|\left(I+\left(B_{D}^{+}\right)^{-1} C_{D}\right)^{-1}\right\|_{\infty}\left\|\left(B_{D}^{+}\right)^{-1}\right\|_{\infty} \leq(n-1)\left\|\left(B_{D}^{+}\right)^{-1}\right\|_{\infty} .
$$

By Lemma 2, we have

$$
\begin{aligned}
\left\|\left(B_{D}^{+}\right)^{-1}\right\|_{\infty} \leq & \max \left\{\sum_{i=1}^{n} \frac{1}{\left(1-d_{i}+b_{i i} d_{i}\right)\left(1-u_{i}\left(B_{D}^{+}\right) p_{i}\left(B_{D}^{+}\right)\right)} \prod_{j=1}^{i-1} \frac{u_{j}\left(\left(B_{D}^{+}\right)\right)}{1-u_{j}\left(\left(B_{D}^{+}\right)\right) p_{j}\left(B_{D}^{+}\right)},\right. \\
& \left.\sum_{i=1}^{n} \frac{p_{i}\left(B_{D}^{+}\right)}{\left(1-d_{i}+b_{i i} d_{i}\right)\left(1-u_{i}\left(\left(B_{D}^{+}\right)\right) p_{i}\left(B_{D}^{+}\right)\right)} \prod_{j=1}^{i-1} \frac{1}{1-u_{j}\left(B_{D}^{+}\right) p_{j}\left(B_{D}^{+}\right)}\right\} .
\end{aligned}
$$

By Lemma 3, we can easily get the following results: for each $i, j, k \in \mathbb{N}$,

$$
\begin{aligned}
b_{k}\left(B_{D}^{+}\right) & =\max _{k+1 \leq i \leq n}\left\{\frac{\sum_{j=k, \neq i}^{n}\left|b_{i j}\right| d_{i}}{1-d_{i}+b_{i i} d_{i}}\right\} \leq \max _{k+1 \leq i \leq n}\left\{\frac{\sum_{j=k, \neq i}^{n}\left|b_{i j}\right|}{b_{i i}}\right\}=b_{k}\left(B^{+}\right), \\
p_{k}\left(B_{D}^{+}\right) & =\max _{k+1 \leq i \leq n}\left\{\frac{\left|b_{i k}\right| d_{i}+\sum_{j=k+1, \neq i}^{n}\left|b_{i j}\right| d_{i} b_{k}\left(B_{D}^{+}\right)}{1-d_{i}+b_{i i} d_{i}}\right\} \\
& \leq \max _{k+1 \leq i \leq n}\left\{\frac{\left|b_{i k}\right|+\sum_{j=k+1, \neq i}^{n}\left|b_{i j}\right| b_{k}\left(B_{D}^{+}\right)}{b_{i i}}\right\} \\
& \leq \max _{k+1 \leq i \leq n}\left\{\frac{\left|b_{i k}\right|+\sum_{j=k+1, \neq i}^{n}\left|b_{i j}\right| b_{k}\left(B^{+}\right)}{b_{i i}}\right\} \\
& =p_{k}\left(B^{+}\right),
\end{aligned}
$$


and

$$
\begin{aligned}
\frac{1}{\left(1-d_{i}+b_{i i} d_{i}\right)\left(1-u_{i}\left(B_{D}^{+}\right) p_{i}\left(B_{D}^{+}\right)\right)} & =\frac{1}{1-d_{i}+b_{i i} d_{i}-\sum_{j=i+1}^{n}\left|b_{i j}\right| d_{i} p_{i}\left(B_{D}^{+}\right)} \\
& \leq \frac{1}{\min \left\{b_{i i}-\sum_{j=i+1}^{n}\left|b_{i j}\right| p_{i}\left(B^{+}\right), 1\right\}} \\
& =\frac{1}{\min \left\{\hat{\beta}_{i}, 1\right\}} .
\end{aligned}
$$

Furthermore, by Lemma 3, we have

$$
\begin{aligned}
\frac{u_{i}\left(B_{D}^{+}\right)}{1-u_{i}\left(B_{D}^{+}\right) p_{i}\left(B_{D}^{+}\right)} & =\frac{\sum_{j=i+1}^{n}\left|b_{i j}\right| d_{i}}{1-d_{i}+b_{i i} d_{i}-\sum_{j=i+1}^{n}\left|b_{i j}\right| d_{i} p_{i}\left(B_{D}^{+}\right)} \\
& \leq \frac{\sum_{j=i+1}^{n}\left|b_{i j}\right|}{b_{i i}-\sum_{j=i+1}^{n}\left|b_{i j}\right| p_{i}\left(B^{+}\right)} \\
& =\frac{1}{\hat{\beta}_{i}} \sum_{j=i+1}^{n}\left|b_{i j}\right|
\end{aligned}
$$

and

$$
\begin{aligned}
\frac{1}{1-u_{i}\left(B_{D}^{+}\right) p_{i}\left(B_{D}^{+}\right)} & =\frac{1-d_{i}+b_{i i} d_{i}}{1-d_{i}+b_{i i} d_{i}-\sum_{j=i+1}^{n}\left|b_{i j}\right| d_{i} p_{i}\left(B_{D}^{+}\right)} \\
& \leq \frac{1-d_{i}+b_{i i} d_{i}}{b_{i i}-\sum_{j=i+1}^{n}\left|b_{i j}\right| p_{i}\left(B^{+}\right)} \\
& =\frac{b_{i i}}{\hat{\beta}_{i}} .
\end{aligned}
$$

By (7), (8), and (9), we obtain

$$
\left\|\left(B_{D}^{+}\right)^{-1}\right\|_{\infty} \leq \max \left\{\sum_{i=1}^{n} \frac{1}{\min \left\{\hat{\beta}_{i}, 1\right\}} \prod_{j=1}^{i-1}\left(\frac{1}{\hat{\beta}_{j}} \sum_{k=j+1}^{n}\left|b_{j k}\right|\right), \sum_{i=1}^{n} \frac{p_{i}\left(B^{+}\right)}{\min \left\{\hat{\beta}_{i}, 1\right\}} \prod_{j=1}^{i-1} \frac{b_{j j}}{\hat{\beta}_{j}}\right\} .
$$

Therefore, the result in (5) holds from (6) and (10).

Since a $B$-matrix is also a $w c d d B$-matrix, then by Theorem 1 , we find the following result.

Corollary 1 Let $M=\left[m_{i j}\right] \in \mathbb{R}^{n \times n}$ be a B-matrix with the form $M=B^{+}+C$, where $B^{+}=\left[b_{i j}\right]$ is defined as (1). Then

$$
\begin{aligned}
& \max _{d \in[0,1]^{n}}\left\|(I-D+D M)^{-1}\right\|_{\infty} \\
& \quad \leq \max \left\{\sum_{i=1}^{n} \frac{n-1}{\min \left\{\hat{\beta}_{i}, 1\right\}} \prod_{j=1}^{i-1}\left(\frac{1}{\hat{\beta}_{j}} \sum_{k=j+1}^{n}\left|b_{j k}\right|\right), \sum_{i=1}^{n} \frac{(n-1) p_{i}\left(B^{+}\right)}{\min \left\{\hat{\beta}_{i}, 1\right\}} \prod_{j=1}^{i-1} \frac{b_{j j}}{\hat{\beta}_{j}}\right\},
\end{aligned}
$$

where $\hat{\beta}_{i}$ is defined as in Theorem 1. 
We next give a comparison of the bounds in (4) and (5) as follows.

Theorem 2 Let $M=\left[m_{i j}\right] \in \mathbb{R}^{n \times n}$ be a wcdd B-matrix with the form $M=B^{+}+C$, where $B^{+}=\left[b_{i j}\right]$ is defined as (1). Let $\bar{\beta}_{i}, \tilde{\beta}_{i}$, and $\hat{\beta}_{i}$ be defined as in (3), (4), and (5), respectively. Then

$$
\begin{aligned}
& \max \left\{\sum_{i=1}^{n} \frac{n-1}{\min \left\{\hat{\beta}_{i}, 1\right\}} \prod_{j=1}^{i-1}\left(\frac{1}{\hat{\beta}_{j}} \sum_{k=j+1}^{n}\left|b_{j k}\right|\right), \sum_{i=1}^{n} \frac{(n-1) p_{i}\left(B^{+}\right)}{\min \left\{\hat{\beta}_{i}, 1\right\}} \prod_{j=1}^{i-1} \frac{b_{j j}}{\hat{\beta}_{j}}\right\} \\
& \quad \leq \sum_{i=1}^{n}\left(\frac{n-1}{\min \left\{\tilde{\beta}_{i}, 1\right\}} \prod_{j=1}^{i-1} \frac{b_{j j}}{\tilde{\beta}_{j}}\right) .
\end{aligned}
$$

Proof Since $B^{+}$is a $w c d d$ matrix with positive diagonal elements, for any $i \in \mathbb{N}$,

$$
0 \leq p_{i}\left(B^{+}\right) \leq 1, \quad \tilde{\beta}_{i} \leq \hat{\beta}_{i} .
$$

By (13), for each $i \in \mathbb{N}$,

$$
\frac{1}{\hat{\beta}_{i}} \leq \frac{1}{\tilde{\beta}_{i}}, \quad \frac{1}{\min \left\{\hat{\beta}_{i}, 1\right\}} \leq \frac{1}{\min \left\{\tilde{\beta}_{i}, 1\right\}} .
$$

The result in (12) follows by (13) and (14).

\section{Remark 1}

(i) Theorem 2 shows that the bound in (5) is better than that in (4).

(ii) When $n$ is very large, one needs more computations to obtain these upper bounds by (5) than by (4).

\section{Numerical examples}

In this section, we present numerical examples to illustrate the advantages of our derived results.

Example 1 Consider the family of $B$-matrices in [14]:

$$
M_{k}=\left[\begin{array}{cccc}
1.5 & 0.5 & 0.4 & 0.5 \\
-0.1 & 1.7 & 0.7 & 0.6 \\
0.8 & -0.1 \frac{k}{k+1} & 1.8 & 0.7 \\
0 & 0.7 & 0.8 & 1.8
\end{array}\right]
$$

where $k \geq 1$. Then $M_{k}=B_{k}^{+}+C_{k}$, where

$$
B_{k}^{+}=\left[\begin{array}{cccc}
1 & 0 & -0.1 & 0 \\
-0.8 & 1 & 0 & -0.1 \\
0 & -0.1 \frac{k}{k+1}-0.8 & 1 & -0.1 \\
-0.8 & -0.1 & 0 & 1
\end{array}\right]
$$

By (2), we have

$$
\max _{d \in[0,1]^{4}}\left\|\left(I-D+D M_{k}\right)^{-1}\right\|_{\infty} \leq \frac{4-1}{\min \{\beta, 1\}}=30(k+1) .
$$


It is obvious that

$$
30(k+1) \rightarrow+\infty, \quad \text { if } k \rightarrow+\infty
$$

By (3), we get

$$
\max _{d \in[0,1]^{4}}\left\|\left(I-D+D M_{k}\right)^{-1}\right\|_{\infty} \leq 15.2675 .
$$

By Theorem 7 of [11], we have

$$
\max _{d \in[0,1]^{4}}\left\|\left(I-D+D M_{k}\right)^{-1}\right\|_{\infty} \leq 13.6777
$$

By Corollary 1 of [13], we have

$$
\max _{d \in[0,1]^{4}}\left\|\left(I-D+D M_{k}\right)^{-1}\right\|_{\infty} \leq \sum_{i=1}^{4}\left(\frac{3}{\min \left\{\tilde{\beta}_{i}, 1\right\}} \prod_{j=1}^{i-1} \frac{b_{j j}}{\tilde{\beta}_{j}}\right) \approx 15.2675
$$

By (11), we obtain

$$
\max _{d \in[0,1]^{4}}\left\|\left(I-D+D M_{k}\right)^{-1}\right\|_{\infty} \leq 9.9683
$$

In these two cases, the bounds in (2) are equal to $60(k=1)$ and $90(k=2)$, respectively.

Example 2 Consider the $w c d d B$-matrix in [13]:

$$
M=\left[\begin{array}{cccc}
1.5 & 0.2 & 0.4 & 0.5 \\
-0.1 & 1.5 & 0.5 & 0.1 \\
0.5 & -0.1 & 1.5 & 0.1 \\
0.4 & 0.4 & 0.8 & 1.8
\end{array}\right]
$$

Then $M=B^{+}+C$, where

$$
B^{+}=\left[\begin{array}{cccc}
1 & -0.3 & -0.1 & 0 \\
-0.6 & 1 & 0 & -0.4 \\
0 & -0.6 & 1 & -0.4 \\
-0.4 & -0.4 & 0 & 1
\end{array}\right] \text {. }
$$

By (4), we get

$$
\max _{d \in[0,1]^{4}}\left\|(I-D+D M)^{-1}\right\|_{\infty} \leq 41.1111
$$

By (5), we have

$$
\max _{d \in[0,1]^{4}}\left\|(I-D+D M)^{-1}\right\|_{\infty} \leq 21.6667 .
$$




\section{Conclusions}

In this paper, we present some new upper bounds for $\max _{d \in[0,1]^{n}}\left\|(I-D+D M)^{-1}\right\|_{\infty}$ when $M$ is a weakly chained diagonally dominant $B$-matrix, which improve some existing results. A numerical example shows that the given bounds are efficient.

\section{Competing interests}

The author declares that he has no competing interests.

\section{Author's contributions}

Only the author contributed to this work. The author read and approved the final manuscript.

\section{Acknowledgements}

The author is grateful to the referees for their useful and constructive suggestions. This work is supported by the National Natural Science Foundation of China $(11361074,11501141)$, the Foundation of Science and Technology Department of Guizhou Province ([2015]7206), the Natural Science Programs of Education Department of Guizhou Province ([2015]420), and the Research Foundation of Guizhou Minzu University (16yjsxm002, 16yjsxm040).

Received: 1 October 2016 Accepted: 16 January 2017 Published online: 02 February 2017

\section{References}

1. Chen, XJ, Xiang, SH: Perturbation bounds of P-matrix linear complementarity problems. SIAM J. Optim. 18, 1250-1265 (2007)

2. Cottle, RW, Pang, JS, Stone, RE: The Linear Complementarity Problem. Academic Press, San Diego (1992)

3. Murty, KG: Linear Complementarity, Linear and Nonlinear Programming. Heldermann Verlag, Berlin (1998)

4. Peña, JM: A class of $P$-matrices with applications to the localization of the eigenvalues of a real matrix. SIAM J. Matrix Anal. Appl. 22, 1027-1037 (2001)

5. Chen, XJ, Xiang, SH: Computation of error bounds for P-matrix linear complementarity problem. Math. Program. 106, 513-525 (2006)

6. Chen, TT, Li, W, Wu, X, Vong, S: Error bounds for linear complementarity problems of MB-matrices. Numer. Algorithms 70(2), 341-356 (2015)

7. Dai, PF, Lu, CJ, Li, YT: New error bounds for the linear complementarity problem with an SB-matrix. Numer. Algorithms 64, 741-757 (2013)

8. García-Esnaola, M, Peña, JM: Error bounds for linear complementarity problems for B-matrices. Appl. Math. Lett. 22, 1071-1075 (2009)

9. García-Esnaola, M, Peña, JM: Error bounds for linear complementarity problems involving $B^{5}$-matrices. Appl. Math. Lett. 25, 1379-1383 (2012)

10. García-Esnaola, M, Peña, JM: B-Nekrasov matrices and error bounds for linear complementarity problems. Numer. Algorithms 72(2), 435-445 (2016)

11. Li, CQ, Gan, MT, Yang, SR: A new error bound for linear complementarity problems for B-matrices. Electron. J. Linear Algebra 31, 476-484 (2016)

12. Shivakumar, PN, Chew, KH: A sufficient condition for nonvanishing of determinants. Proc. Am. Math. Soc. 43(1), 63-66 (1974)

13. Li, CQ, Li, YT: Weakly chained diagonally dominant B-matrices and error bounds for linear complementarity problems. Numer. Algorithms 73(4), 985-998 (2016)

14. Li, CQ, Li, YT: Note on error bounds for linear complementarity problems for B-matrices. Appl. Math. Lett. 57, 108-113 (2016)

15. Huang, TZ, Zhu, Y: Estimation of $\left\|A^{-1}\right\|_{\infty}$ for weakly chained diagonally dominant M-matrices. Linear Algebra Appl. 432, 670-677 (2010)

\section{Submit your manuscript to a SpringerOpen ${ }^{\circ}$ journal and benefit from:}

- Convenient online submission

Rigorous peer review

- Immediate publication on acceptance

- Open access: articles freely available online

- High visibility within the field

- Retaining the copyright to your article 